\title{
Quantum magneto-transport in two-dimensional GaAs electron gases and SiGe hole gases
}

\author{
C.-T. Liang ${ }^{a, *}$, Yu-Ming Cheng ${ }^{a}$, Tsai Yu Huang ${ }^{\text {a }}$, C.F. Huang ${ }^{a}$, M.Y. Simmons ${ }^{\text {b,c }}$, \\ D.A. Ritchie ${ }^{b}$, Gil-Ho Kim ${ }^{\text {d }}$ J.Y. Leem ${ }^{\mathrm{e}}$, Y.H. Chang ${ }^{\mathrm{a}}$, Y.F. Chen ${ }^{\mathrm{a}}$ \\ ${ }^{a}$ Department of Physics, National Taiwan University, Taipei 106, Taiwan \\ ${ }^{\mathrm{b}}$ Cavendish Laboratory, Madingley Road, Cambridge CB3 OHE, UK \\ ${ }^{\mathrm{c}}$ Semiconductor Nanofabrication Facilities, School of Physics, University of New South Wales, Sydney 2052, Australia \\ ${ }^{\mathrm{d}}$ Telecommunication Basic Research Laboratory, ETRI, Yusong PO Box 106, Taejon 305-600, South Korea \\ ${ }^{\mathrm{e}}$ Materials Evaluation Centre, Korea Research Institute of Standards and Sciences (KRISS), PO Box 102, Taejon 305-600, South Korea
}

Received 20 December 2000; accepted 26 December 2000

\begin{abstract}
We have measured the low-temperature transport properties of two-dimensional (2D) GaAs electron gases and 2D SiGe hole gases. Our experimental results fall into three categories. (i) Collapse of spin-splitting and an enhanced Landé $g$-factor at Landau level filling factors both $\nu=3$ and $\nu=1$ in a 2D GaAs electron gas are observed. Our experimental results show direct evidence that the effective disorder is stronger at $\nu=1$ than that at $\nu=3$ over approximately the same perpendicular magnetic field range. (ii) We present evidence for spin-polarisation of a dilute 2D GaAs electron gas. The Lande $g$-factor of the system is estimated to be 1.66. This enhanced $g$ value is ascribed to electron-electron interactions at ultra low carrier density limit. (iii) In a high-quality SiGe hole gas, there is a temperature-independent point in the magnetoresistivity $\rho_{x x}$ and $\rho_{x y}$ which is ascribed to experimental evidence for a quantum phase transition between $\nu=3$ and $\nu=5$. We also present a study on the temperature $(T)$ driven flow lines in our system. (C) 2001 Elsevier Science Ltd. All rights reserved.
\end{abstract}

Keywords: A. Semiconductors; B. Epitaxial growth; B. Vapour deposition; D. Transport properties

\section{Introduction}

Two-dimensional (2D) systems based on semiconductor devices play an indispensable role in our daily lives. For example, the silicon ( $\mathrm{Si}$ ) metal-oxide-semiconductor field-effect transistor (MOSFET) is the most important device for microprocessor and memories in our computers. GaAs high-electron-mobility-transistors (HEMTs) have been widely used in satellite communications and mobile phones. More recently, advances in technology have made it possible to grow high-quality 2D SiGe hole systems. Given the present and potential application of GaAs and SiGe structures, such 2D structures are not only important to everyday lives, but also provide ideal systems for studying exciting physics.

\footnotetext{
* Corresponding author.

E-mail address: ctliang@phys.ntu.edu.tw (C.-T. Liang).
}

In this paper, we present low-temperature measurements on 2D GaAs electron gases and SiGe hole gases. The structure of this paper is as follows. Section 2 describes activation studies on a GaAs electron gas at Landau level filling factors $\nu=3$ and $\nu=1$. Section 3 presents spindependent transport in a 2D GaAs electron gas in a parallel magnetic field. Magneto-transport measurements on SiGe hole gases are presented in Section 4. In Section 5 we summarise our experimental results, together with some conclusions.

\section{Activation studies at $\nu=1$ and $\nu=3$}

When a large magnetic field is applied perpendicular to the plane of a low-disordered 2D electron gas (2DEG), the 2DEG exhibits the integer quantum Hall effect [1] at liquid helium temperatures. The picture of extended states at the Landau level centres and localised states between Landau 
levels provides a simple description of the quantum Hall effect in a strong perpendicular magnetic field.

It is now well established that the energy gap $\Delta_{\nu}$ at a Landau level filling factor can be determined from the exponential temperature dependence of magnetoresistivity $\rho_{x x} \approx$ $\exp \left(-\Delta_{\nu} / 2 \mathrm{k}_{\mathrm{B}} T\right)$, where $\mathrm{k}_{\mathrm{B}}$ is the Boltzmann constant and $T$ is the temperature, respectively. This approach is valid in both the integer and fractional quantum Hall regimes [2-4]. At $\nu=1, \Delta_{1}$ is simply the 'spin gap' which has the form [5]

$\Delta_{1}=g_{0} \mu_{\mathrm{B}} B+E_{\mathrm{ex}}=g^{*} \mu_{\mathrm{B}} B$,

where $E_{\mathrm{ex}}$ is the many-body exchange energy which lifts the $g$-factor from its bare value $\left(g_{0}=0.44\right)$ to its enhanced value $g^{*}, \mu_{\mathrm{B}}$ is the Bohr magneton and $B$ is the applied magnetic field. This spin gap approach is also valid for other oddnumber filling factors, for example, $\nu=3$.

The front-gated Hall bar used in this work was made from GaAs/ $/ \mathrm{Al}_{0.3} \mathrm{Ga}_{0.7}$ As heterostructures. At $V_{g}=0 \mathrm{~V}$, the carrier density of the 2 DEG is $3.3 \times 10^{15} \mathrm{~m}^{-2}$ with a mobility of $30 \mathrm{~m}^{2} \mathrm{~V}^{-1} \mathrm{~s}^{-1}$, without illumination. Measurements were performed in a top-loading ${ }^{3} \mathrm{He}$ cryostat using standard four-terminal ac phase sensitive techniques.

Fig. 1 shows an activation plot $\ln \rho_{x x}(\nu=1)$ as a function of $1 / T$ at various carrier densities. From the straight line fits shown in Fig.1, we can measure $\Delta_{1}$ at different carrier densities, and Fig. 2 shows such results. It is evident that $\Delta_{1}$ shows a linear dependence on $B$ (and hence $n_{\mathrm{s}}$ ), as demonstrated by the straight line fit through the full squares. According to Eq. (1), we know that the exchange energy $E_{e x}$ is approximately linear in $B$ in our system. The measured spin gap is also enhanced over the single-particle Zeeman energy which is shown in the dotted line. From the linear fit shown in the solid line, we estimate $g^{*}$ to be 3.11 and a critical magnetic field $B_{\mathrm{c}}$ of $1.5 \mathrm{~T}$ in which $\Delta_{1}$ collapses to zero. An interception of $-1.31 \mathrm{~K}$ at the $y$-axis is ascribed to disorder broadening at $\nu=1$ in our case. All our experimental results are consistent with the work by Kim et al. [5], in which InAs was inserted into the centre of the GaAs quantum well. In our system, at low $B$ the data (labelled as open squares) show slight deviation from the straight line fit, suggesting that the actual critical field is higher than $B_{\mathrm{c}}$ determined from the linear fit.

In the previous work of Kim et al. [5], due to the moderate disorder within the InAs/GaAs systems, the minimum in $\rho_{x x}$ at $\nu=3$ was not well resolved. Our GaAs system is of higher quality and we are able to study the spin gap at $\nu=3$. Fig. 3 shows $\ln \rho_{x x}(\nu=3)$ as a function of $1 / T$ at various carrier densities. The spin gaps at $\nu=3$ are determined from the straight line fits shown in Fig. 3. The measured spin gap $\Delta_{3}$ is also enhanced over the single-particle Zeeman energy, as clearly shown in Fig. 4. From the slope of the linear fit, we estimate the $g^{*}$ to be 4.05 . It is evident that the data at $\nu=3$ are similar to that at $\nu=1$ : both collapse of spin-splitting and the enhanced $g^{*}$ over the bare value are observed. The measured $g^{*}{ }_{\nu=3}=4.05$ is larger

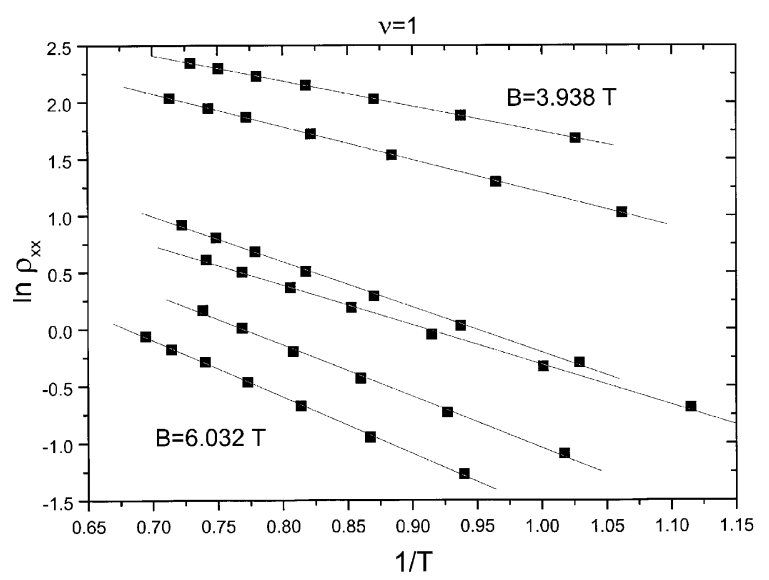

Fig. 1. The logarithm of $\rho_{x x}(\nu=1)$ versus the inverse of temperature $1 / T$ at different gate voltages (and hence magnetic fields $B$ ). From top to bottom: $B=3.938,4.262,4.65,5.076,5.592$ and $6.032 \mathrm{~T}$. The slopes of the straight line fits $\Delta_{1}$ are shown in Fig. 2.

than $g^{*}{ }_{\nu=1}=3.11$, showing direct evidence that manybody interactions are stronger at $\nu=3$ than those at $\nu=1$. The fact that the magnitudes of the critical field $B_{\mathrm{c}} \approx 0.8 \mathrm{~T}$ and an interception of $-0.8 \mathrm{~K}$ at $\nu=3$ are both smaller than those at $\nu=1$ also shows that the effective disorder at $\nu=1$ is larger than that at $\nu=3$ over approximately the same measurement range $4 \mathrm{~T} \leq B \leq 6 \mathrm{~T}$.

\section{Spin-dependent transport in a parallel magnetic field}

In a low-dimensional electron system [6], applying an in-plane magnetic field $B_{\|}$parallel to the plane of the electron gas has been proved to be a powerful technique to study spin-dependent electron transport. The first tilted magnetic field experiment on a 2DEG revealed an enhancement of the $g$-factor [7]. In a one-dimensional (1D) electron system, it was first demonstrated by Wharam et al. [8] that a large $B_{\|}$lifts the electron spin-degeneracy, causing consecutive spin-parallel (parallel to $B_{\|}$) and spin-antiparallel (antiparallel to $B_{\|}$) conductance plateaux in multiples of $e^{2} / h$ [9]. Using a source-drain bias technique [10], Patel et al. measured the Landé $g$-factor in a one-dimensional (1D) constriction for the first time [9]. Later, this measurement was extended to the case of an ultra-high-quality $1 \mathrm{D}$ electron gas. When the $1 \mathrm{D}$ channel is wide, it is found that the measured Landé $g$-factor is $\approx 0.4$, close to that of bulk GaAs. As the channel is progressively narrowed, there is an enhancement of the Landé $g$-factor [11].

Recently, there has been a great deal of interest in the metal-insulator transition (MIT) in dilute 2D systems in 


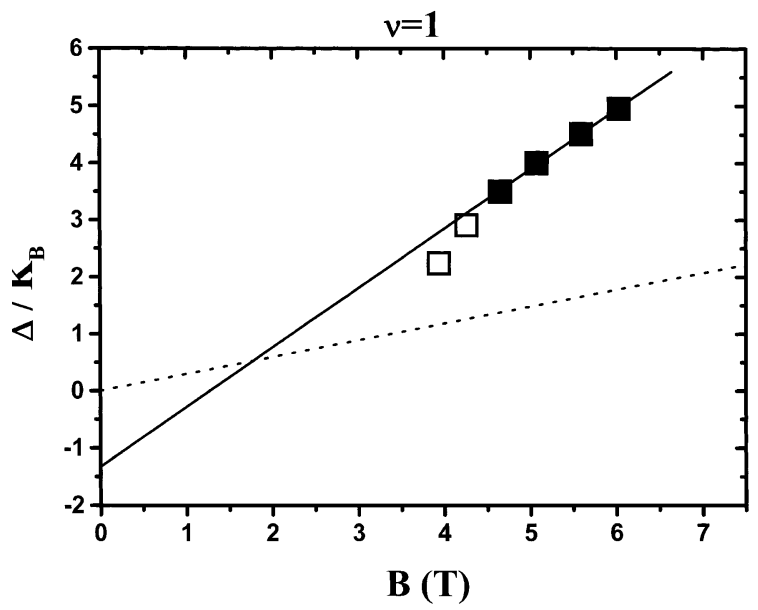

Fig. 2. The experimentally determined $\Delta_{1}$ at various magnetic fields $B$. The straight line fit is discussed in the text. The dotted line is the bare Zeeman energy assuming $g_{0}=0.44$.

an in-plane magnetic field [12-16]. It was suggested that the observed strong magnetoresistance in high in-plane fields is a manifestation of the spin alignment of free carriers [15]. The suppression of the 'metallic state' with increasing in-plane magnetic field has now become important in trying to understand the underlying physics of the 'metal-insulator transition' in two dimensions.

Previously we studied spin-dependent electron transport in a quasiballistic quantum wire [17]. It was found that at zero split-gate voltage when the quasiballistic wire is not electrostatically defined, the two-terminal conductance due to the bulk 2DEG shows monotonic decrease with increasing $B_{\|}$. In order to gain more insights into the effect of a $B_{\|}$on 2 DEG transport and exclude any contact resistance effect, we measure the four-terminal magnetoresistivity of a gated 2D electron system at various carrier densities. In this section, we report low-temperature magnetoresistivity measurements of a dilute 2D GaAs electron gas. Using a simple model, we estimate the Landé $g$-factor in this dilute 2DEG to be about 1.66. The enhanced value of the Landé $g$-factor in this dilute limit compared with that of a bulk 2DEG (0.44) is ascribed to electron-elecron interactions.

The measurements were performed on a gated Hall bar made from $\mathrm{GaAs} / \mathrm{Al}_{0.33} \mathrm{Ga}_{0.67} \mathrm{As}$ heterostructure. At $V_{g}=0$, the carrier concentration of the $2 \mathrm{DEG}$ was $1.53 \times 10^{11} \mathrm{~cm}^{-2}$ with a mobility of $4 \times 10^{6} \mathrm{~cm}^{2} \mathrm{~V}^{-1} \mathrm{~s}^{-1}$ after brief illumination by a red light emitting diode. The depth of the 2DEG is $300 \mathrm{~nm}$ for our device. Experiments were performed in a top-loading ${ }^{3} \mathrm{He}$ cryostat at $T=300 \mathrm{mK}$ and the four-terminal magnetoresistivity was measured with standard phase-sensitive techniques. The in-plane magnetic $B_{\|}$is applied parallel to the sourcedrain current. To check for an out-of-plane magnetic field component, we measure the Hall voltage. From this, we know that the sample was aligned better than $0.1^{\circ}$ using an in situ rotating insert.

We now turn to our main experimental results. Fig. 5 shows the four-terminal magnetoresistivity $\rho_{x x}$ as a function of in-plane magnetic field $B_{\|}$at various carrier densities $n_{\mathrm{s}}$. Let us consider the uppermost curve. It is evident that $\rho_{x x}$ shows a $B_{\|}^{2}$ dependence for $B_{\|}<5 \mathrm{~T}$ and shows a weaker $B_{\|}^{2}$ dependence for $B_{\|}>9 \mathrm{~T}$, as shown by the two dotted lines. We ascribe the increase in $\rho_{x x}$ at low $B_{\|}$to gradual spin alignment of the 2DEG $[15,16]$. The physical origin of $B_{\|}^{2}$ at high $B_{\|}$is not understood, but we speculate that it is due to the enhancement of electron scattering when the magnetic length is comparable to the thickness of the 2DEG. To obtain quantitative information on this spin alignment effect, we use an empirical method similar to those reported $[15,16]$, but using two parabolic fits, as shown in the two dotted lines in Fig. 5 for various $n_{\mathrm{s}}$. The interception of parabolic fits is defined as the 'crossing field' $B_{\text {cross }}$ for a certain 2D carrier density. As shown later, from $B_{\text {cross }}\left(n_{\mathrm{s}}\right)$ we can estimate the $g$-factor in our system. Note that the magnetoresistivity shows little temperature dependence between $T=0.3 \mathrm{~K}$ and $T=1 \mathrm{~K}$, suggesting that the $B_{\|}^{2}$ dependence is a semi-classical effect. Also, within our experimental accuracy, we did not observe a 'metal-insulator transition' in our device. The reason for this is probably due to the rather weak MIT behaviour in a 2D GaAs electron gas [18] and the limited measurement temperature range in our system.

Fig. 6 shows the crossing field $B_{\text {cross }}$ as a function of both carrier concentration $n_{\mathrm{s}}$ and the corresponding local Fermi energy $E$. Following the previous work $[15,16]$, we assume the slope of the $E-B_{\text {cross }}$ diagram is given by the Zeeman

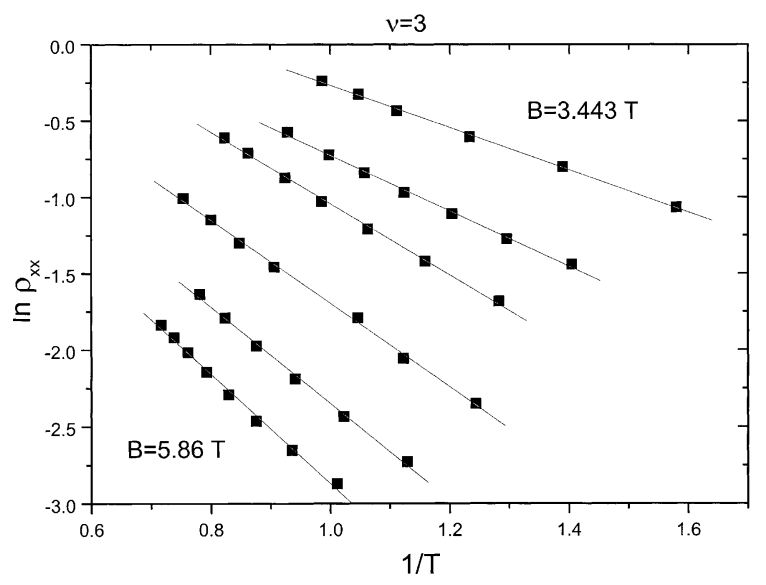

Fig. 3. The logarithm of $\rho_{x x}(\nu=3)$ versus the inverse of temperature $1 / T$ at different gate voltages (and hence magnetic fields $B$ ). From top to bottom: $B=3.443,3.818,4.064,4.667,5.263$ and $5.860 \mathrm{~T}$. The slopes of the straight line fits $\Delta_{1}$ are shown in Fig. 4. 


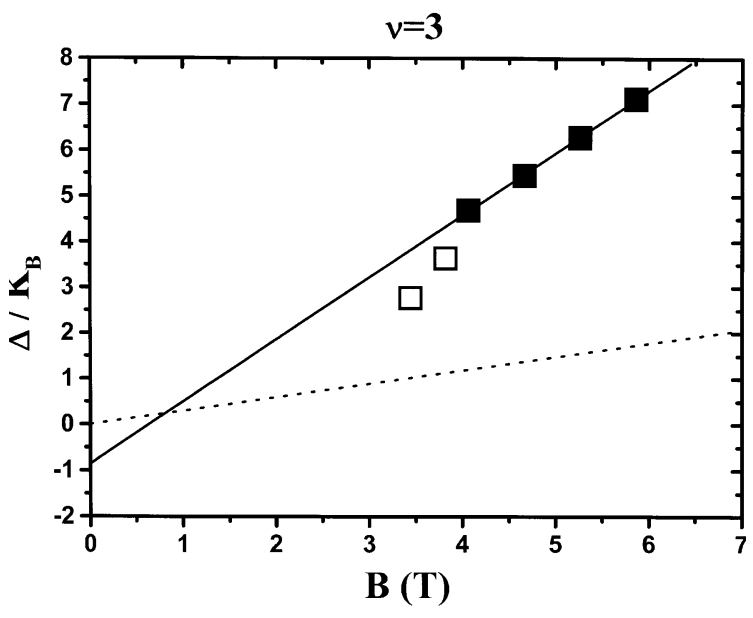

Fig. 4. The experimentally determined $\Delta_{3}$ at various magnetic fields $B$. The straight line fit is discussed in the text. The dotted line is the bare Zeeman energy assuming $g_{0}=0.44$.

energy $E=g \mu_{B} B_{\|}$. In this case, a linear fit through the origin gives an estimated $g$-factor of 1.42. As shown in Fig. 3, the best linear fit yields a value of the $g$-factor of 1.66. This fit gives a negative interception at $B=0$ which can be attributed to disorder broadening [16]. Note that both measured values are close to that measured in a clean 1D electron gas when there is a single 1D subband occupied [11]. Previously, this enhancement of $g$-factor [11] was ascribed to electron-electron interactions at low carrier densities.

It is well known that the application of an in-plane magnetic field causes a diamagnetic shift in the 2D conduction edge, giving rise to an energy shift proportional to $B_{\|}^{2}$. This effect is believed to cause shifts in single electron tunnelling peak positions in back-gate voltage in a quantum dot [19] and the pinch-off voltage of a split-gate device [11] with increasing $B_{\|}$. However, in previous work on a dilute 2D GaAs hole gas [15], in measurements on high-mobility Si MOSFETs [16], and in this present work, no clear evidence of energy shift with $B_{\|}^{2}$ due to the diamagnetic effect is found. Thus, we speculate that in dilute $2 \mathrm{D}$ systems, the Zeeman effect is much more pronounced than the diamagnetic effect.

We would like to address the issue of a 1D electron gas in a parallel magnetic field. Experimentally, a 1D electron gas is connected to bulk $2 \mathrm{D}$ electron reservoirs $[8,11,17]$. It is generally assumed that the application of an in-plane magnetic field does not fully spin-polarise the bulk 2DEG. In this case, the applied $B_{\|}$only lifts the electron spindegeneracy in the $1 \mathrm{D}$ region. From the estimated enhanced $g$-factor in our experiment at $B_{\|}=12 \mathrm{~T}$ the Zeeman energy is given by $1.15 \mathrm{meV}$, which is still considerably smaller than the Fermi energy $E_{\mathrm{F}}$ in the bulk $2 \mathrm{DEG}(10.5 \mathrm{meV}$ for $n_{\mathrm{s}}=3 \times 10^{11} \mathrm{~cm}^{-2}$ [17] and $7 \mathrm{meV}$ for $n_{\mathrm{s}}=2 \times 10^{11} \mathrm{~cm}^{-2}$ [11]). Thus, it is safe to assume that the bulk $2 \mathrm{DEG}$ is spindegenerate at a high $B_{\|}$. However, as the $2 \mathrm{DEG}$ carrier density is decreased to $\approx 4 \times 10^{10} \mathrm{~cm}^{-2}$, one has to be careful when studying the in-plane magnetic field dependence of a $1 \mathrm{D}$ electron system connected to bulk 2DEG regions, since at high enough $B_{\|} \approx 12 \mathrm{~T}$, the $2 \mathrm{DEG}$ regions can also be fully spin-polarised.

\section{Measurements on a SiGe hole gas}

The SiGe alloy is technically important for its potential high-performance device application. Recent advances in crystal growth have made it possible to fabricate unprecedented high-quality $\mathrm{Si} / \mathrm{SiGe}$ hole gas samples. Since then, the magneto-transport properties in a SiGe hole gas have been attracting a great deal of experimental and theoretical interest. For example, at a Landau level filling factor $\nu=1.5$, an insulating phase observed in SiGe hole samples [20-23] is not fully understood at present. It is called a 'Hall insulator', since although $\rho_{x x}$ approaches infinity when the temperature $T \rightarrow 0$, the Hall resistivity remains finite at approximately $h / 2 e^{2}$. Most noticeably, this magneto-driven quantum phase transition, unanticipated by the global phase diagram proposed by Kivelson et al. [24] has become a subject of intensive studies.

Early theoretical work on the integer quantum Hall effect described a two-parameter scaling [25] in which both the dissipative conductance $\sigma_{x x}$ and the Hall conductance $\sigma_{x y}$ vary with sample length $L$. Note that the renormalisation-group function can be illustrated by flow lines [25]. After successive length-scale transformation, the flow lines are directed towards fixed points $\left(\sigma_{x x}, \sigma_{x y}\right)=(0, n)$ in which $n=0,1,2, \ldots$ is the Landau level index. In addition to these 'localisation fixed points' which describe localisation of wave functions

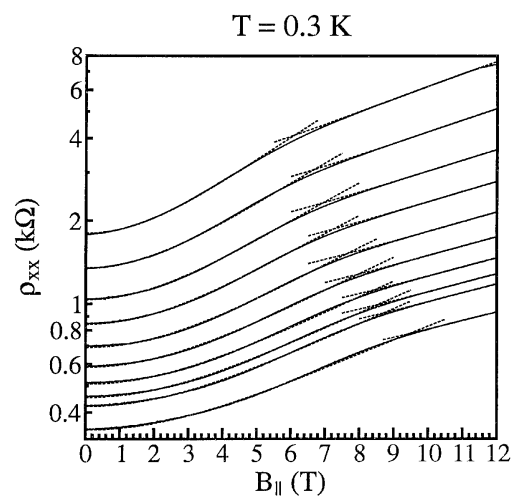

Fig. 5. $\rho_{x x}\left(B_{\|}\right)$for various carrier densities. From top to bottom: $n_{\mathrm{s}}=1.379,1.481,1.591,1.688,1.780,1.884,1.967,2.036,2.076$ and $2.226 \times 10^{10} \mathrm{~cm}^{-2}$, respectively. Two parabolic fits for $B_{\|}<5 \mathrm{~T}$ and $B_{\|}>9 \mathrm{~T}$ for various $n_{\mathrm{s}}$ are shown in dotted lines. 


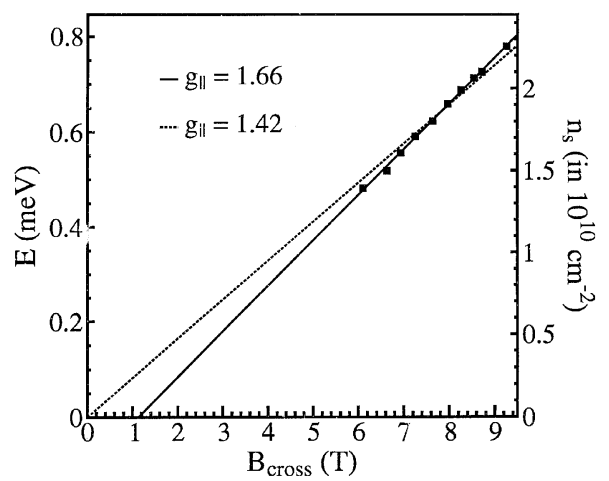

Fig. 6. Local Fermi energy $E$ and the corresponding 2D carrier density $n_{\mathrm{s}}$ at various measured crossing fields $B_{\text {cross. }}$ The straight line fit through the origin is shown in the dotted line. The best linear fit is shown in the solid line.

of electrons near the Fermi energy, there are intermediate-coupling fixed points on $\sigma_{x y}=n+1 / 2$. Note that this theory is developed for $T=0$ and the length scale transformations are accomplished by varying the sample length $L$. It has been shown that, in practice, the effective sample size can be varied by changing the temperature [26]. This 'temperature-driven flowlines' study supports the two-parameter scaling theory by Pruisken [25].

In this section, we report low-temperature magneto-transport properties of a two-dimensional SiGe hole gas. There is a temperature-independent point in $\rho_{x x}$ and $\rho_{x y}$ which is ascribed to experimental evidence for a quantum phase transition between Landau level filling factors $\nu=3$ and $\nu=5$. Finally, we present a study on the temperature-driven flow lines in our system.

The Hall bar used in this work was made from a highquality $\mathrm{Si} / \mathrm{Si}_{0.88} \mathrm{Ge}_{0.12}$ heterostructure which was grown in a multi-wafer UHV/CHD system. In our structure, holes are confined in two dimensions in a SiGe layer of $\approx 40 \mathrm{~nm}$ thick, sandwiched between the $\mathrm{Si}$ substrate and cap layer. The carrier concentration of the $2 \mathrm{D}$ hole gas (2DHG) is $3.3 \times 10^{11} \mathrm{~cm}^{-2}$ with a mobility of $\approx 600 \mathrm{~cm}^{2} \mathrm{~V}^{-1} \mathrm{~s}^{-1}$. The experiments were performed in a top-loading ${ }^{3} \mathrm{He}$ cryostat. Four-terminal magnetoresistivities were measured using standard phase-sensitive lock-in techniques.

Previous transport measurements on SiGe hole gas samples were mostly undertaken in the 'Hall insulator' regime. In this section, we describe measurements at low magnetic fields. The detailed temperature dependence of $\rho_{x x}$ and $\rho_{x y}$ are shown in Fig. 7(a) and (b). At zero magnetic field, there is a small decrease in $\rho_{x x}$ as the temperature is lowered, characteristic of the metallic phase close to the metal-insulator transition [27,28]. An interesting feature of Fig. 7(a) and (b) is that there is a point which is temperature-independent around a critical magnetic field $B_{\mathrm{cr}} \approx 3 \mathrm{~T}$ for both $\rho_{x x}$ and $\rho_{x y}$ between Landau level filling factors $\nu=5$ and $\nu=3$ (marked by the vertical dash line in Fig. 7). The $\rho_{x x}$ maximum is not centred around a particular $B$ value, but becomes increasingly displaced to higher $B$ as the temperature is increased. We convert the data to conductivities using matrix inversion, and the results are shown in Fig. 7(c). The temperature-independent conductivities are $\sigma_{x x} \approx 2 e^{2} / h$ and $\sigma_{x y} \approx 3 e^{2} / h$, respectively. We suggest that the temperature-independent point in $\rho_{x x}$, $\rho_{x y}, \sigma_{x x}$ and $\sigma_{x y}$ corresponds to a quantum-phase transition boundary. We note that similar behaviour was observed between adjacent quantum Hall states at $\nu=3-2$ and $\nu=2-1$ transitions in a higher quality SiGe hole gas [29].

Previous temperature-driven flow lines study was performed on GaAs electron gases in the regime where the minima in $\rho_{x x}$ at even Landau level filling factors are observed. In a SiGe hole gas, the Zeeman splitting is close to that of Landau splitting [7], so that minima in $\rho_{x x}$ are observed at odd-number Landau filling factors, as clearly shown in Fig. 7. Thus, it is interesting to study the $T$-driven flow lines in a SiGe hole gas. Fig. 8 shows experimental $\sigma_{x x}(T)$ and $\sigma_{x y}(T)$ plotted as $T$-driven flow lines from $T=0.3$ to $1.648 \mathrm{~K}$. Each line corresponds to a fixed $B$. We can see that an intermediate-coupling point as labelled as squares. To the right of this point, the flow lines direct towards $(0,4.5)$, since the Hall conductance plateau at $\nu=5$ is not well quantised. In contrast to the semi-circle-like feature seen by Wei and co-workers [26], to the left of the intermediate-coupling point, the $T$-driven flow lines direct towards $\sigma_{x y}=2.0$ and eventually converge at $\sigma_{x y}=3.0$. This effect is believed to be due to strong Landau level mixing as described by the model of Ando [30]. The intermediate-coupling point is close to $\sigma_{x y}=3.25$, not the theoretical value $\sigma_{x y}=3.5$ as predicted by Pruisken [25]. We note that early experimental results by Wei [26] indeed showed similar behaviour.

\section{Conclusions}

In conclusion, we have measured the low-temperature transport properties of 2D GaAs electron gases and 2D SiGe hole gases. In particular, (i) in a GaAs electron gas, we find an enhancement of the $g$-factors and collapse of spin splitting at both Landau level filling factors $\nu=3$ and $\nu=1$. The enhanced $g$-factors are ascribed to many-body exchange interactions. We also provide evidence that the electron-electron interactions at $\nu=3$ are stronger than those at $\nu=1$. (ii) $\mathrm{We}$ present experimental evidence for spin-polarisation of a dilute GaAs electron gas in a parallel magnetic field. The in-plane $g$-factor is estimated to be 1.66 . This enhanced value over the bare $g$-factor in bulk 


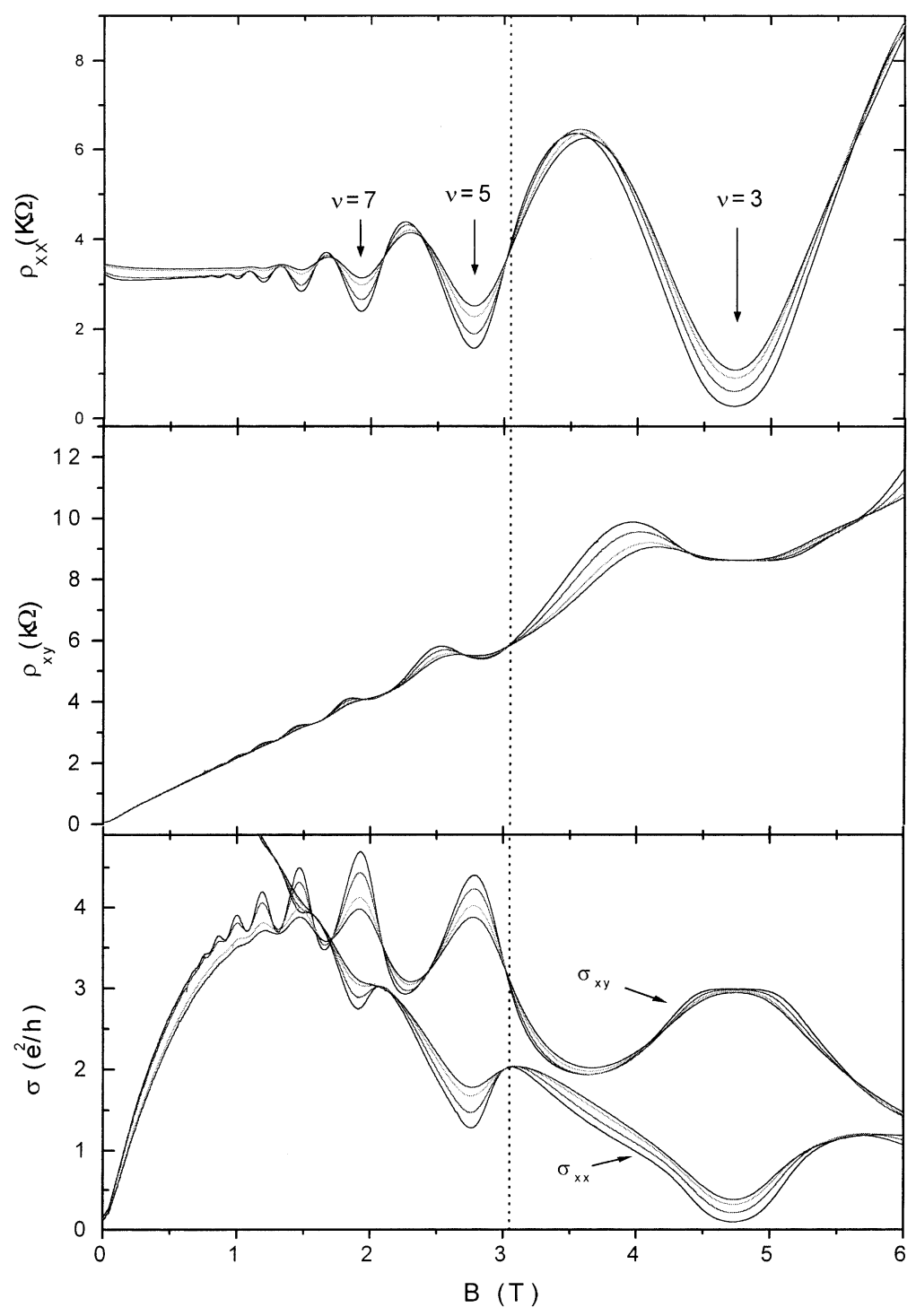

Fig. 7. (a) $\rho_{x x}(B)$, (b) $\rho_{x y}(B)$ and (c) $\sigma_{x x}(B)$ and $\sigma_{x y}(B)$ of a SiGe hole gas sample at various temperatures $T$. From top to bottom: $T=0.3,0.6$, 0.924 and $1.2 \mathrm{~K}$. The dotted line illustrates a temperature-independent point in $\rho_{x x}(B), \rho_{x y}, \sigma_{x x}$ and $\sigma_{x y}$.

GaAs (0.44) is due to electron-electron interactions at ultra-low carrier densities. (iii) In a SiGe hole gas, there is a temperature-independent point in $\rho_{x x}$ and $\rho_{x y}$, which is ascribed to experimental evidence for a quantum phase transition between Landau level filling factors $\nu=3$ and $\nu=5$. Finally, we present a study on the temperature $(T)$-driven flow lines in $\sigma_{x x}(T)$ and $\sigma_{x y}(T)$. The behaviour of the flow lines is believed to be due to strong Landau-level mixing in our system. In summary, our new experimental results suggest that there is unexpected and exciting physics in GaAs and SiGe two-dimensional systems. Thus, we are confident that there will be more striking results coming out of this fertile area of research in the near future.

\section{Acknowledgements}

This work was funded by the NSC, Taiwan (grant nos. NSC 89-2112-M-002-052, 89-2112-002-084 and NSC 89-2911-I-002-104). The work at Cambridge University was supported by the EPSRC, UK. The 


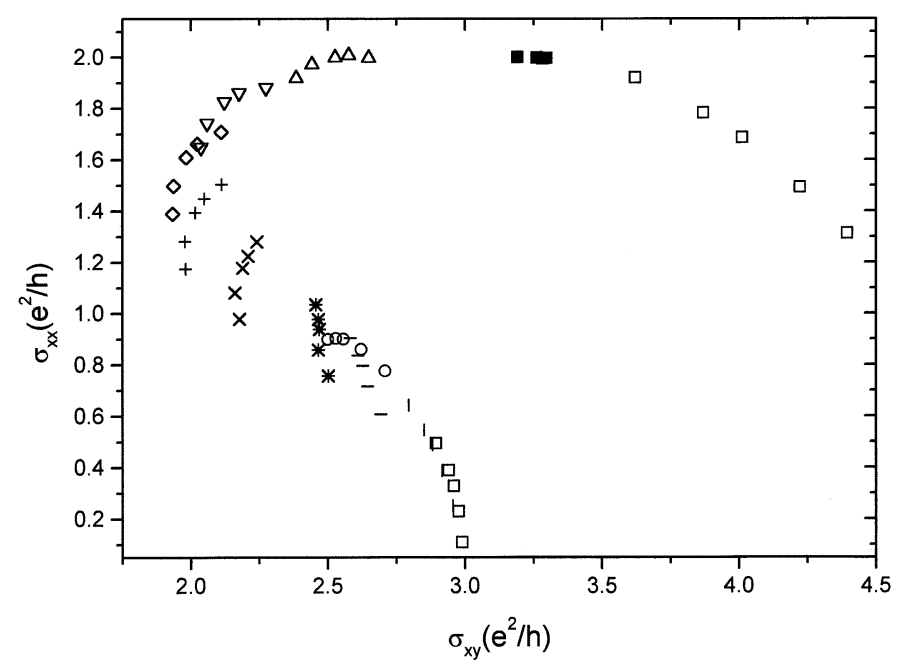

Fig. 8. Experimental $\sigma_{x x}(T)$ and $\sigma_{x y}(T)$ plotted as $T$-driven flow lines from $T=0.3$ to $1.648 \mathrm{~K}$. Each symbol corresponds to a fixed $B$.

low-temperature measurements were performed at the high-magnetic field facilities at the Centre for Condensed Matter Sciences (CCMS), National Taiwan University (NTU). We thank H.H. Cheng and T.J. Chuang at the CCMS for support, and R.R. Du, R.B. Dunford, F.F. Fang and C.G. Smith, for useful discussions. C.T.L. acknowledges financial support from the Department of Physics, NTU. M.Y.S. is grateful for support from the ARC, Australia. G.H.K. acknowledges financial support from the Korean Ministry of Information and Communication.

\section{References}

[1] K. von Klitzing, G. Dorda, M. Pepper, Phys. Rev. Lett. 45 (1980) 449-452.

[2] R.R. Du, H.L. Stormer, D.C. Tsui, L.N. Pfeiffer, K.W. West, Phys. Rev. Lett. 70 (1993) 2944-2947.

[3] D.R. Leadley, R.J. Nicholas, C.T. Foxon, J.J. Harris, Phys. Rev. Lett. 72 (1994) 1906-1909.

[4] R.R. Du, H.L. Stormer, D.C. Tsui, L.N. Pfeiffer, K.W. West, Solid State Commun. 90 (1994) 71-74.

[5] G.H. Kim, J.T. Nicholls, S.I. Khondaker, I. Farrer, D.A. Ritchie, Phys. Rev. B 61 (2000) 19010-19016.

[6] A.B. Fowler, F.F. Fang, W.E. Howard, P.J. Stiles, Phys. Rev. Lett. 16 (1966) 901-904.

[7] F.F. Fang, P.J. Stiles, Phys. Rev. 174 (1968) 823-825.

[8] D.A. Wharam, T.J. Thornton, R. Newbury, M. Pepper, H. Ahmed, J.E.F. Frost, D.G. Hasko, D.C. Peacock, D.A. Ritchie, G.A.C. Jones, J. Phys. C 21 (1988) L209-L214.

[9] N.K. Patel, J.T. Nicholls, L. Martin-Moreno, M. Pepper, J.E.F. Frost, D.A. Ritchie, G.A.C. Jones, Phys. Rev. B 44 (1991) 10973-10975.
[10] N.K. Patel, J.T. Nicholls, L. Martin-Moreno, M. Pepper, J.E.F. Frost, D.A. Ritchie, G.A.C. Jones, Phys. Rev. B 44 (1991) 13549-13555.

[11] K.J. Thomas, J.T. Nicholls, M. Pepper, M.Y. Simmons, D.R. Mace, D.A. Ritchie, Phys. Rev. Lett. 77 (1996) 136139.

[12] D. Simonian, S.V. Kravchenko, M.P. Sarachik, V.M. Pudalov, Phys. Rev. Lett. 79 (1997) 2304-2307.

[13] M.Y. Simmons, A.R. Hamilton, M. Pepper, E.H. Linfield, P.D. Rose, D.A. Ritchie, A.K. Savchenko, T.G. Griffiths, Phys. Rev. Lett. 80 (1998) 1292-1295.

[14] T. Okamoto, K. Hosoya, S. Kawaji, A. Yagi, Phys. Rev. Lett. 82 (1999) 3875-3878.

[15] J. Yoon, C.C. Li, D. Shahar, D.C. Tsui, M. Shaygen, Phys. Rev. Lett. 84 (2000) 4421-4424.

[16] V.M. Pudalov, G. Brunthaler, A. Prinz, G. Bauer, Cond-mat/ 0004206 (2000).

[17] C.-T. Liang, M. Pepper, M.Y. Simmons, C.G. Smith, D.A. Ritchie, Phys. Rev. B 61 (2000) 9952-9955.

[18] Y. Hanein, U. Meirav, D. Shahar, C.C. Li, D.C. Tsui, H. Shtrikman, Phys. Rev. B 58 (1998) 13338-13340.

[19] J. Weis, R.J. Haug, K. von Klitzing, K. Ploog, Surf. Sci. 305 (1994) 664-668.

[20] F.F. Fang, P.J. Wang, B.S. Meyerson, J.J. Nocera, K.E. Ismail, Surf. Sci. 263 (1992) 175-178.

[21] R.B. Dunford, E.E. Mitchell, R.G. Clark, V.A. Stadnik, F.F. Fang, R. Newbury, R.H. McKenzie, R.P. Starrett, P.J. Wang, B.S. Meyerson, J. Phys. Condens. Matter 9 (1997) 15651570.

[22] P.T. Coleridge, A.S. Sachrajda, P. Zawadzki, R.L. Williams, H. Lofontaine, Solid State Commun. 10 (1997) 755-760.

[23] T.Y. Lin, M.S. Tsai, Y.F. Chen, F.F. Fang, J. Phys. Condens. Matter 10 (1998) 9691-9698.

[24] S. Kivelson, D.H. Lee, S.C. Zhang, Phys. Rev. B 46 (1992) 2223-2245. 
[25] A.M.M. Pruisken, Phys. Rev. B 32 (1985) 2636-2639.

[26] H.P. Wei, D.C. Tsui, A.A.M. Pruisken, Phys. Rev. B 33 (1985) 1488-1490.

[27] J. Lam, M. D’Iorio, D. Brown, H. Lafontaine, Phys. Rev. B 56 (1997) 12741-12744.
[28] P.T. Coleridge, R.L. Williams, Y. Feng, P. Zawadzki, Phys. Rev. B 56 (1997) 12764-12767.

[29] R.B. Dunford, N. Griffin, M. Pepper, P.J. Phillips, T.E. Whall, Physica E 6 (2000) 297-300.

[30] T. Ando, J. Phys. Soc. Japan 55 (1986) 3199-3209. 